\title{
Gradhiva
}

GRADHIV

Revue d'anthropologie et d'histoire des arts

$14 \mid 2011$

Carl Einstein et les primitivismes

\section{Art, agentivité et collectivité}

Art, Agency and Collectivity

\section{Charles W. Haxthausen}

Traducteur : Isabelle Kalinowski

\section{CpenEdition}

\section{Journals}

Édition électronique

URL : http://journals.openedition.org/gradhiva/2159

DOI : 10.4000/gradhiva.2159

ISSN : 1760-849X

Éditeur

Musée du quai Branly Jacques Chirac

Édition imprimée

Date de publication : 30 novembre 2011

Pagination : 78-99

ISBN : 978-2-35744-046-3

ISSN : 0764-8928

\section{Référence électronique}

Charles W. Haxthausen, «Art, agentivité et collectivité », Gradhiva [En ligne], 14 | 2011, mis en ligne le

30 novembre 2014, consulté le 02 mai 2019. URL : http://journals.openedition.org/gradhiva/2159 ;

DOl : 10.4000/gradhiva.2159 


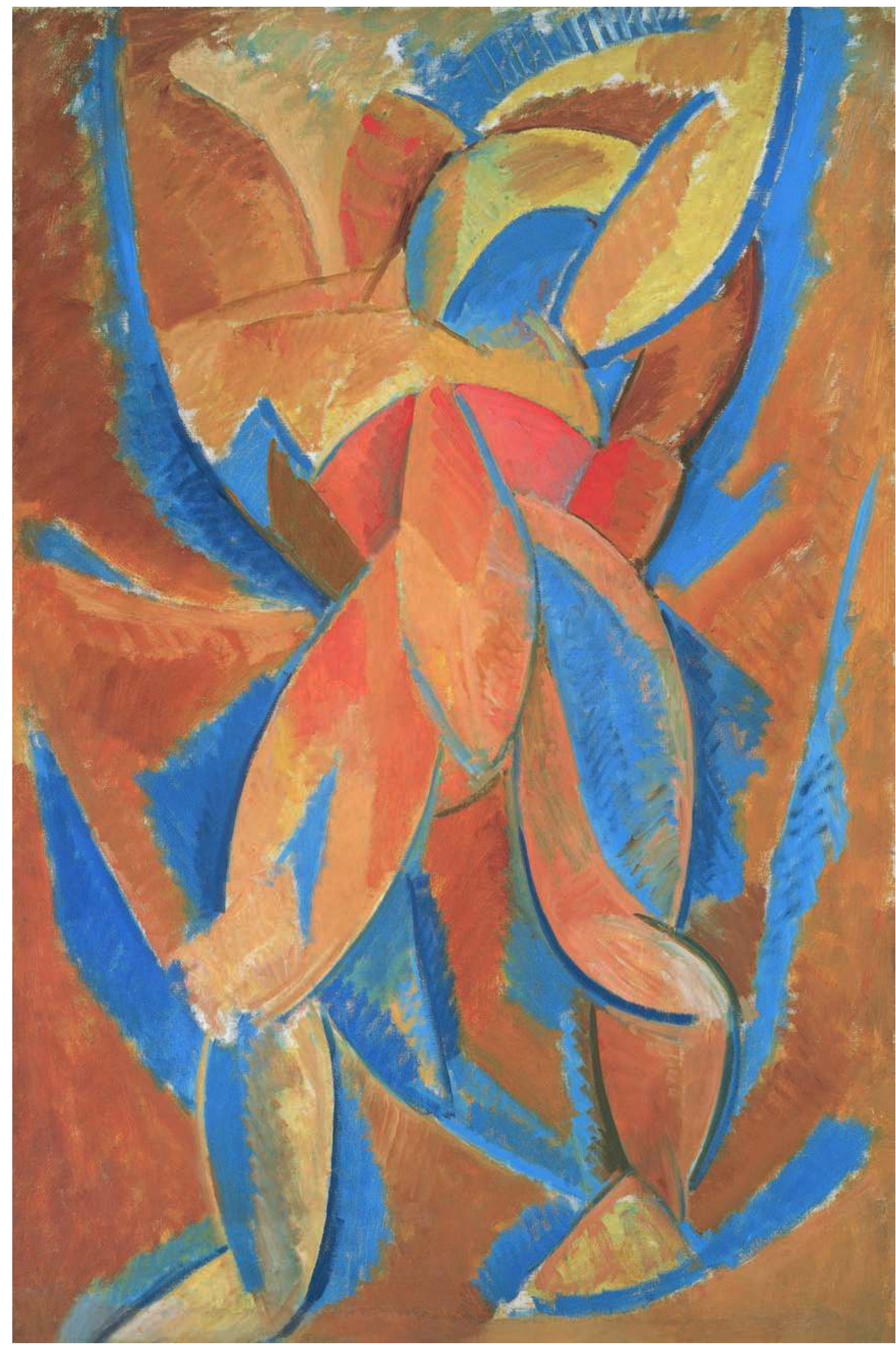

Fig. 1 Pablo Picasso, Nu debout, 1908, exposé à la Neue Galerie, Berlin, décembre 1913. Museum of Fine Arts, Boston. DR / Scala, Florence @ Succession Picasso, 2011. 
Charles W. Haxthausen

\begin{abstract}
$\bullet \bullet$
1. Marianne Kröger a utilisé cette formule dans le titre du chapitre introductif de son livre, Das "Individuum als Fossil". Carl Einsteins Romanfragment "BEB II": das Verhältnis von Autobiographie, Kunst und Politik in einem Avantgardeprojekt zwischen Weimarer Republik und Exil, Remscheid, Gardez! Verlag, 2007, p. $13-90$
\end{abstract}

2. Mentionnons des exceptions dans les années d'avant-guerre : «Anmerkungen [1912; «Notes 》), «Politische Anmerkungen [1912; « Notes politiques 》), «Der Arme (1913; «Le pauvre 》) et «Die Sozialdemokratie [1914; « La socialdémocratie »), in Carl Einstein, Werke, t. I (1907-1918], Hermann Haarmann et Klaus Siebenhaar [éd.], Berlin, Fannei \& Walz, 1994, p. 142-146, 156-159, 213.

3. Carl Einstein, Die Fabrikation der Fiktionen, Sibylle Penkert (éd.) Reinbek bei Hamburg, Rowohlt, 1973.

\section{Art et agentivité et agentivité
collectivité}

Si la vie de Carl Einstein peut à juste titre être décrite comme " une vie entre l'art et la politique", la relation entre ces deux termes reste à préciser ${ }^{1}$. Caractériser cette vie comme partagée entre art et politique suggère d'emblée une distance, une tension entre ces deux univers; de fait, d'un point de vue chronologique, ces deux pôles furent généralement associés à des épisodes bien distincts. Lorsque Einstein s'engagea dans l'activité politique - durant la révolution allemande avortée de 1918-1919 et la guerre civile espagnole de 1936-1939-, il ne publia qu'un court texte sur l'art. Inversement, dans les périodes où il se consacra essentiellement à ses publications sur l'art, comme en 1913-1914 et de 1921 à 1933, il ne publia presque aucun texte sur la politique, du moins sur un mode explicite ${ }^{2}$. Jusqu'au milieu des années 1930, Einstein fit rarement le lien entre ces deux domaines dans son écriture. Le texte dans lequel ils sont articulés de la façon la plus décisive, le manuscrit La Fabrication des fictions, de la taille d'un livre, ne fut pas publié de son vivant ${ }^{3}$.

Il serait cependant erroné de penser à un divorce entre les textes d'Einstein sur l'art et ses textes politiques, même si les premiers sont rarement ouvertement politiques. L'argument le plus parlant que l'on peut sans doute invoquer pour attester qu'il croyait, ardemment, à une telle relation entre art et politique est une autocritique dévastatrice datant de 1934: «L'éternel pseudo-révolutionnaire devient complètement stérile et reste désespérément à la traîne des changements qui affectent son temps parce qu'il lutte toujours pour la même utopie révolutionnaire qu'il tente d'atteindre par un changement de la forme artistique " (c'est l'auteur qui souligne). Cet amer retour sur lui-même est énoncé dans les notes et fragments laissés par Einstein pour Bebuquin II, son roman inachevé, pour une part autobiographique, dans un passage où il évoquait son activisme 


\section{CARL EINSTEIN ET LES PRIMITIVISMES}

\section{-}

4. Ce passage apparaît dans un feuillet de correction placé dans une enveloppe contenant deux feuillets du même type datés de janvier ou février 1934 : «Bebuquin II », Carl-Einstein-Archiv (CEA), microforme 43, feuillet de correction 21, Académie des sciences, Berlin.

5. Carl Einstein, Georges Braque, in id., Werke, t. III (1929-1940), Hermann Haarmann et Klaus Siebenhaar (éd.), Berlin, Fannei \& Walz, 1996, p. 270. Traduction française publiée sous la direction de Liliane Meffre, trad. Jean-Loup Korzilius, Bruxelles, La Part de l'CEil, 2003, p. 32 (traduction modifiée par I. Kalinowski). Bien que le livre n'ait été publié qu'en 1934, le frontispice indique qu'il était achevé en 1932

6. Cf. John Richardson, A Life of Picasso: vol. II, 1907-1917, en collaboration avec Marilyn McCully, New York, Random House, 1996, p. 317, n. 61 et 62. Selon Heike Neumeister, Einstein fut à n'en pas douter l'organisateur de l'exposition; Heike M. Neumeister, « Notes on the "ethnographic turn" of the European avant-garde: Reading Carl Einstein's Negerplastik and Vladimir Markov's Iskusstvo negrov », Acta Historium Artium 49, 2008, p. 174, 175.

7. Carl Einstein, Werke, t. I, op. cit., p. 214-221.

8. Id., Negerplastik, Leipzig, Verlag der weißen Bücher, 1915 ; repris in id., Werke, t. I, op. cit., p. 234-252. Trad. fr. La Sculpture nègre de Liliane Meffre, Paris, L'Harmattan, 1998.

9. Id., Werke, t. I, op. cit., p. 214. politique durant la révolution allemande de 1918-19194. Ce verdict sévère, à l'évidence, était une réflexion rétrospective de 1934; en 1932 encore, dans son Georges Braque, Einstein n'avait pas perdu sa confiance utopique dans l'agentivité sociale radicale de la forme artistique. Le cubisme, déclarait-il, «a créé une vision primaire, il a transformé le pouvoir et la structure de la vision et il a redéfini l'image optique du monde [...]; c'est l'action et le devenir humain qui ont eux-mêmes été

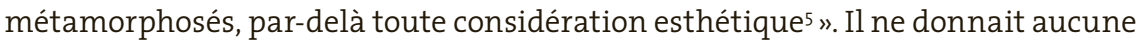
preuve à l'appui de cette affirmation.

Tout en faisant référence de manière récurrente à la "collectivité », Einstein restait vague quant à la nature précise de "l'utopie révolutionnaire" à laquelle la forme artistique était susceptible de donner le jour. En quel sens l'art pouvaitil réaliser cette utopie? Plus précisément, comment la forme artistique était-elle susceptible de générer des formes sociales et politiques particulières - puisque telle était, semble-t-il, la croyance d'Einstein? Un monde visuel collectif formé par l'art et manifesté dans un style unifié pouvait-il faire naître une collectivité sociale égalitaire? Sur toutes ces questions, il demeurait vague. On peut être tenté de penser que c'est justement ce flou qui lui permit de préserver sa croyance et sa vision d'espoir pendant deux décennies. Il ne clarifia ce point qu'en abandonnant la conviction qui avait été la sienne. Dans ce bref essai, je me propose de retracer les étapes majeures de cette odyssée.

En décembre 1913, la Neue Galerie de Berlin, ouverte depuis peu, présenta ce qui était pour l'époque une exposition remarquable et provocatrice : cinquante-trois tableaux, cubistes pour la plupart, et trente dessins de Pablo Picasso, associés à dix-neuf sculptures africaines. Einstein, qui avait alors vingt-huit ans, prit à coup sûr connaissance de cette installation; il en fut peut-être même le maître d'œuvre ${ }^{6}$. Durant les mois qui suivirent, il écrivit deux textes pour lesquels l'exposition de la Neue Galerie avait certainement joué un rôle de catalyseur. Le premier, publié dans trois livraisons successives de la revue politique de gauche Die Aktion, était un essai théorique dense et hermétique sur ce qu'Einstein appelait le principe de «totalité 7 ». Le second, écrit à peu près à la même époque mais qui ne fut publié qu'en 1915, était un livre qui fit date, Negerplastik, le premier ouvrage jamais publié dans lequel la sculpture de l'Afrique subsaharienne était traitée avant tout comme un art ${ }^{8}$.

La notion d'agentivité, telle qu'elle s'exprime dans l'un et dans l'autre, peut permettre de nouer un lien visible entre ces deux textes par ailleurs très différents. Ce qui comptait dans l'art, du point de vue d'Einstein, ce n'était pas sa valeur esthétique, symbolique ou expressive, et moins encore son sujet. C'était sa capacité à renouveler les représentations visuelles des sujets humains et, par là, à transformer leur subjectivité et leur construction de la réalité. Cette idée est développée au début de l'essai «Totalité»:

Par-delà la position spécifique qui est la sienne, l’art détermine la vision en général. [...] L'art transforme la vision globale, et l'artiste infléchit les représentations visuelles en général. Par suite, la finalité de l'art est d'organiser ces derniers. [...] L'art devient une force agissante dès lors qu'il est en mesure d'ordonner la vision en fonction de ses lois. [...] L'objet de l'art ne réside pas dans des objets mais dans la mise en forme de la vision. [...] L'acte de connaissance, c'est-à-dire la transformation de la représentation du monde, n'intervient pas dans la création ni dans la contemplation de l'œuvre d'art; il est le fait de l'œuvre d'art elle-même9. 


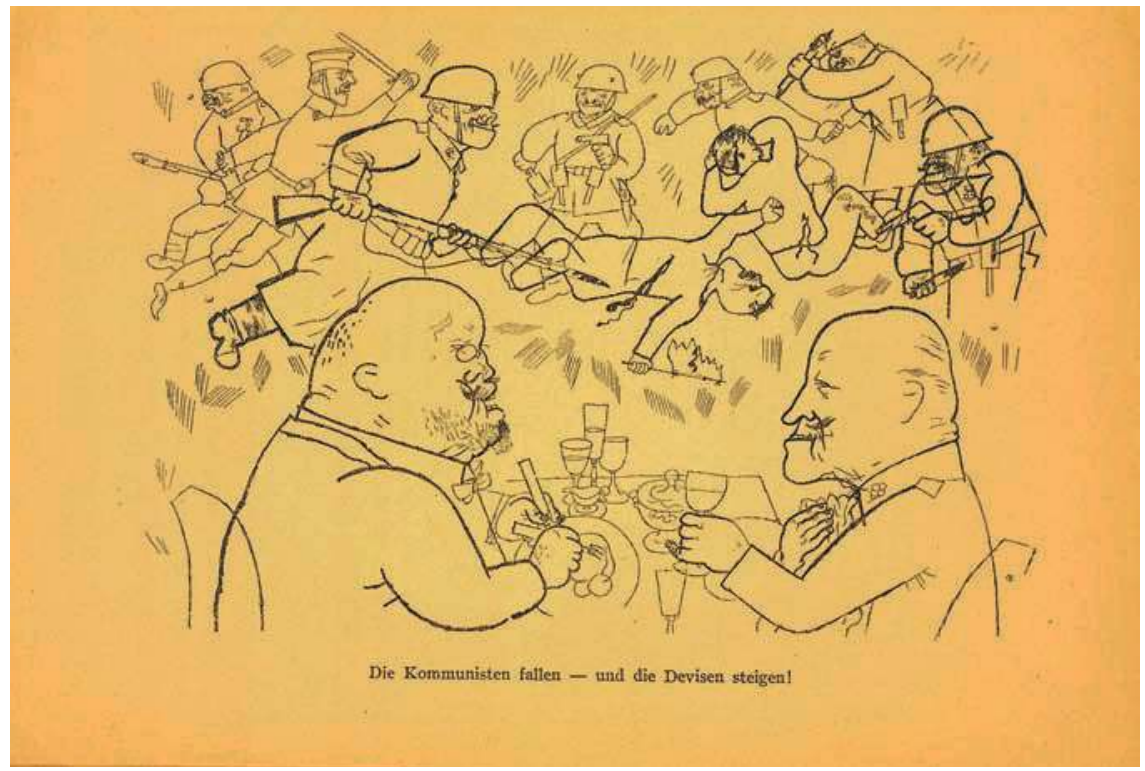

Fig. 2 George Grosz, "Les communistes tombent et les devises augmentent », 1920. BPK, Berlin, dist. RMN / Photographe inconnu @ ADAGP, Paris 2011.

Pour accéder à cette agentivité radicale, l'œuvre d'art doit confronter le spectateur à une "totalité", un tout autosuffisant et formellement autonome, qui interdit toute participation active du spectateur et rend même impossibles les opérations de la mémoire visuelle. La première partie de l'essai, d'où ces observations sont tirées, fut publiée sous le titre «Anmerkungen " ( Notes»), mais, dans une ébauche manuscrite, Einstein les intitula, de façon significative, "Picasso", bien que ni ce dernier ni aucun autre artiste ne fût mentionné dans le texte ${ }^{10}$. Il est clair qu'Einstein pensait avoir trouvé dans le cubisme un art capable de satisfaire ces exigences précises.

Einstein était parfaitement conscient que, à l'exception notable du cubisme, l'art européen de l'époque ne correspondait pas à l'idéal décrit dans l'essai «Totalité "; dans Negerplastik, cependant, il expliquait qu'il s'était réalisé dans l'art africain. La sculpture africaine était un art «inconditionnellement autonome et fermé». Son agentivité tenait à des propriétés formelles et spatiales qui étaient entièrement subordonnées à sa fonction dans la religion africaine. Pour le spectateur africain, l'œuvre d'art n'était pas «une création arbitraire et artificielle» mais «une réalité mythique, dont la force excédait celle de la réalité naturelle ». Elle n'était pas saisie comme une représentation mais comme un être: «Elle ne signifie rien, elle n'est pas symbole; elle est le dieu qui conserve sa réalité mythique close, dans laquelle il inclut l'adorateur, le transforme lui aussi en être mythique et abolit son existence humaine ${ }^{11}$.» Cette idée selon laquelle l'œuvre d'art transformait le spectateur par la négation de sa subjectivité individuelle était appelée à occuper une place centrale dans la théorie einsteinienne de l'agentivité transformatrice de l'art ${ }^{12}$.

La théorie de l'art d'Einstein présente certaines affinités frappantes avec l'œuvre de l'anthropologue britannique Alfred Gell sur l'art et l'agentivité. En élaborant sa «théorie anthropologique de l'art », Gell fut amené à " refuser d'aborder celui-ci en termes de symboles et de significations "; au lieu de mettre l'accent sur "la communication symbolique», il se focalisa sur «l'agentivité, l'intention, le rapport de cause à effet, le résultat et la transformation ». Il regardait l'art « comme
0

10. Carl Einstein, «Picasso 》, CEA, microforme 296, p. 1.

11. Carl Einstein, Werke, t. I, op. cit., p. 242, 246. Trad. fr. p. 31.

12. Cette idée est clairement articulée dans le chapitre final de Negerplastik, «Maske und Verwandtes » [《Masques et apparentés »]. Les masques et les tatouages, observait Einstein, constituent une forme d'autoabnégation au bénéfice du collectif. 


\section{CARL EINSTEIN ET LES PRIMITIVISMES}

-

13. Alfred Gell, Art and Agency: An Anthropological Theory, Oxford-New York, Clarendon Press, 1998, p. 6. Trad. fr. L'Art et ses agents, trad. S. et 0 . Renaut, Dijon, Les Presses du Réel, 2009, p. 8.

14. Carl Einstein, Georges Braque, in Werke, t. III, op. cit., p. 256; trad. fr., op. cit., p. 15 (traduction légèrement modifiée).

15. Alfred Gell, op. cit., p. 7 ; trad. fr. p. 9.

16. Ibid., p. 250. Sur la «personne disséminée », voir p. 96-104; trad fr. p. 299.

17. Sur les déplacements et les activités d'Einstein dans l'armée allemande pendant la guerre, voir Liliane Meffre, Carl Einstein, 1885-1940. Itinéraires d'une pensée moderne, Paris, Presses de l'université Paris-Sorbonne, 2002, p. 58-79.

18. Sur l'engagement révolutionnaire d'Einstein en Belgique, voir Klaus H. Kiefer, « Carl Einstein and the Revolutionary Soldiers' Councils in Brussels », in The Ideological Crisis of Expressionism: the Literary and Artistic German War Colony in Belgium, 1914-1918, Rainer Rumold et O.K. Werckmeiser (éd.], Columbia, Camden House, 1990 p. 97-112.

19. Sur les activités politiques d'Einstein en 1918-1919, voir Dirk Heißerer, «Einsteins Verhaftung: Materialien zum Scheitern eines revolutionären Programms in Berlin und Bayern, 1919 », Archiv für die Geschichte des Widerstandes und der Arbeit 12, 1992, p. 41-77 ; voir aussi Klaus H. Kiefer, Diskurswandel im Werk Carl Einsteins: ein Beitrag zur Theorie und Geschichte der europäischen Avantgarde, Tübingen, Niemeyer, coll. Communicatio ?, 1994, p. 234-255.

20. Carl Einstein, «An die Geistigen » (1919), Werke, t. II (1919-1928), Hermann Haarmann et Klaus Siebenhaar (éd.), Berlin, Fannei \& Walz, 1996, p. 18. un système d'action visant à changer le monde plutôt qu'à codifier des propositions symboliques à son sujet ${ }^{13}$ ». Quoique historien de l'art et critique, Einstein adopta une position étonnamment semblable en déclarant que les œuvres d'art n'avaient d'intérêt "que dans la mesure où elles offraient des moyens de transformer le réel, la structure de l'homme et les images du monde $\mathrm{e}^{14}$ ».

J'ai pour ma part trouvé le livre de Gell particulièrement utile pour clarifier les origines d'une tension - une tension fatale - à l'œuvre dans la théorie einsteinienne de l'agentivité de l'art. L'animisme de la sculpture africaine est parfaitement approprié pour illustrer ce que Gell décrit lui-même comme une "proposition apparemment radicale", à savoir l'idée que, d'un point de vue anthropologique, «les objets d'art sont les équivalents de personnes ou, plus précisément, d'agents sociaux ${ }^{15}$ " (c'est l'auteur qui souligne). Néanmoins, la théorie de Gell est étendue à l'art dans son ensemble, et ne s'applique pas exclusivement à celui qui est produit par des groupes qui croient, sur un mode animiste, à l'agentivité de leurs objets d'art. Se référant à l'œuvre de Marcel Duchamp comme exemple de l'art profane moderne, Gell observe que, à travers ses œuvres d'art et ses écrits, Duchamp devient une "personne disséminée " (distributed person): sa " conscience, le flux de son être en tant qu'agent, n'est pas seulement "accessible pour nous", mais il s'est réalisé sous cette forme. Duchamp s'est tout simplement transformé en cet objet, et il se manifeste à présent partout dans le monde, sous d'innombrables formes, en tant que ces parties détachées de sa personne, ces idoles, ces peaux ou ces objets précieux ${ }^{16}$. " L'agentivité n'est ici pas la même que celle des cultures "primitives", dans lesquelles le "personnage " qui agit à travers l'œuvre d'art n'est pas l'artiste, mais l'esprit d'un ancêtre, une divinité, etc. Les artefacts de ce type sont l'objet central de l'analyse de Gell. La spécificité de la théorie einsteinienne de l'agentivité, nous allons le voir, tient au fait qu'Einstein commença à la fin des années 1920 et au début des années 1930 à appliquer ces notions animistes à certaines œuvres du modernisme européen.

$\mathrm{Au}$ moment de la parution de Negerplastik, en 1915, Einstein était sous les drapeaux, et il passa la plus grande partie de la guerre dans la Belgique occupée par $l^{\prime}$ Allemagne ${ }^{17}$. Même s'il avait publié plusieurs essais politiques avant la guerre, ce n'est qu'en Belgique qu'il s'engagea dans un activisme politique radical. Dans les derniers temps du conflit, des soldats et des ouvriers allemands, épuisés par les années de guerre et inspirés par l'exemple de la révolution d'Octobre en Russie, mirent sur pied des conseils (Räte) partout en Allemagne et en Belgique ${ }^{18}$. De retour à Berlin, Einstein devint un membre très en vue du mouvement des conseils (Rätebewegung), et il fit partie du groupe des six orateurs triés sur le volet qui prirent la parole lors des funérailles de la cofondatrice du Parti communiste allemand, Rosa Luxemburg, après son assassinat ${ }^{19}$. Dans un appel aux intellectuels d'Allemagne, Einstein proclama son adhésion sans équivoque à la révolution : "Une chose importe : la réalisation de la communauté communiste. [...] Nous marchons dans la masse, aux côtés des gens simples, des inconditionnels, nous sommes en marche vers une cause proche et nécessaire... C'en est fini de l'individualisme, seuls comptent les liens avec les camarades de la masse ${ }^{20} . "$

"Sur l'art primitif» [traduit dans ce numéro], qui fut sa première publication sur l'art après Negerplastik, est le seul texte d'Einstein sur l'art qui date de cette période révolutionnaire. Il était pleinement en accord avec les dispositions politiques de son auteur. L'«art primitif» dont il était question dans ce bref article n'était pas l'art africain, mais un art européen issu de la classe ouvrière et placé 


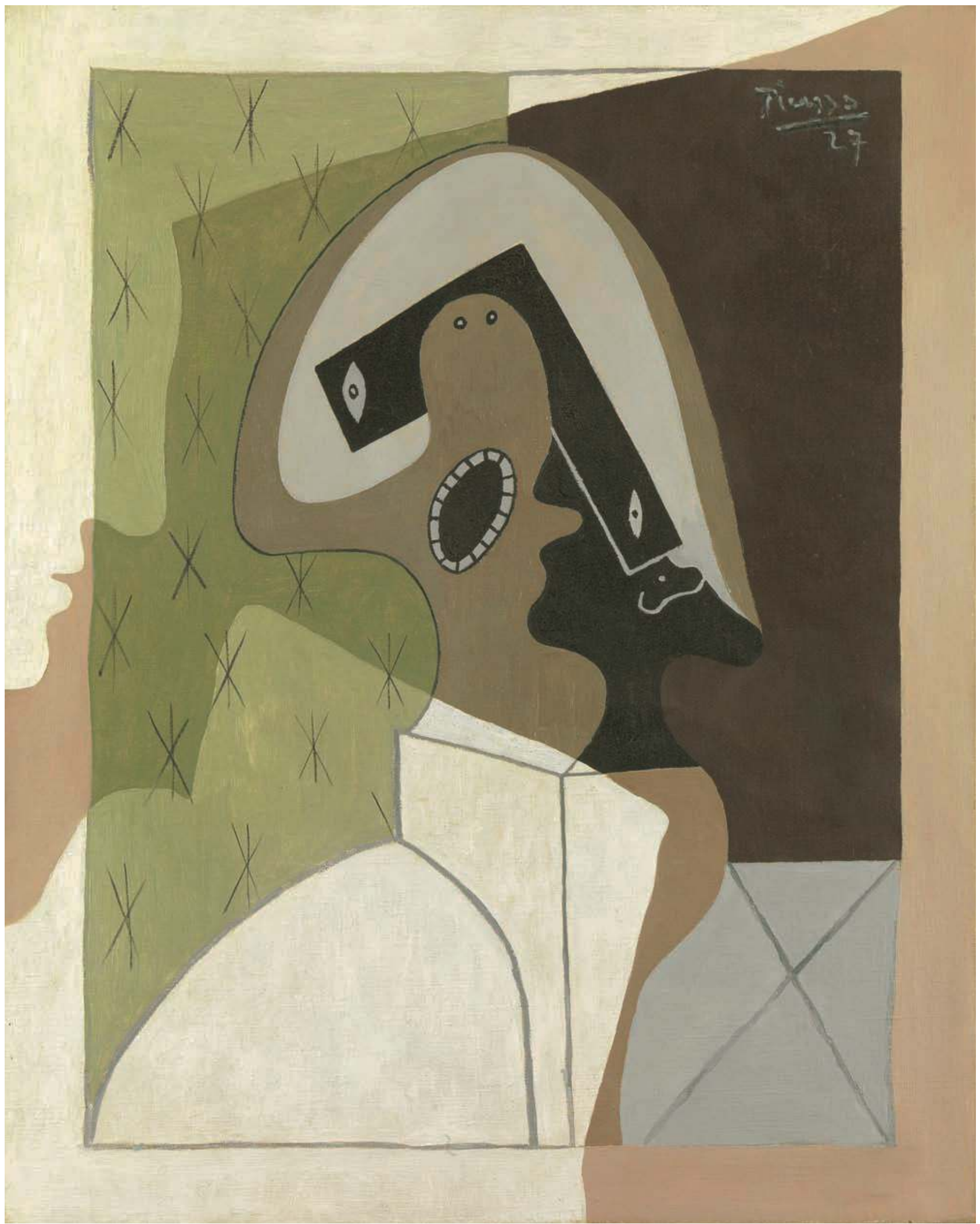

Fig. 3 Pablo Picasso, Arlequin, 1927, reproduit dans Die Kunst des 20. Jahrhunderts. The Metropolitan Museum of Art, dist. RMN / image of the MMA (c) Succession Picasso, 2011. 


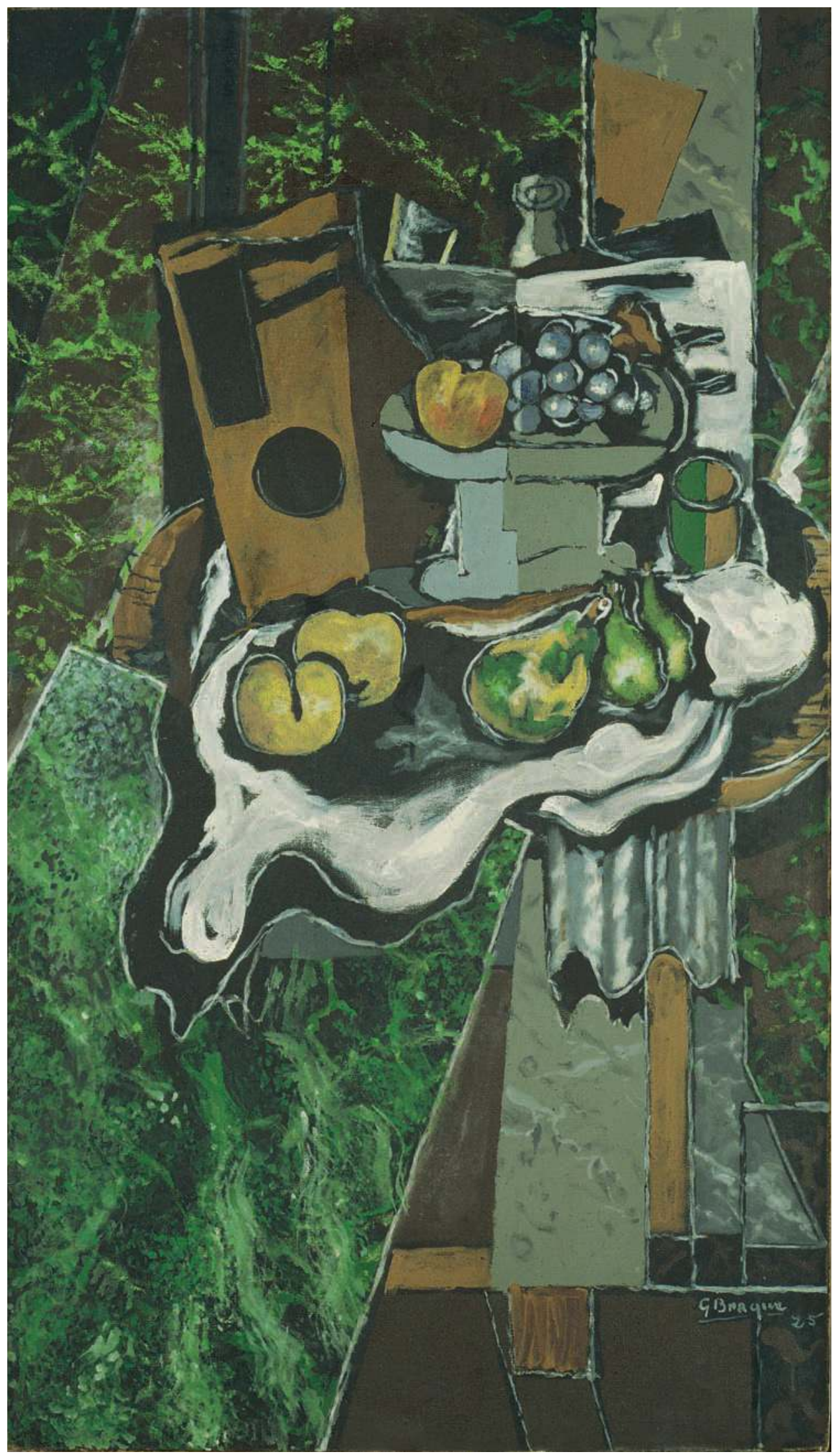

Fig. 4 Georges Braque, Fruits sur une nappe et compotier, 1925 , reproduit dans Georges Braque, 1934. Collection Centre Pompidou, dist. RMN / DR @ ADAGP, Paris 2011. 
au service de la révolution communiste. "Primitif» connotait dès lors la simplicité, le caractère direct et la pauvreté matérielle, non corrompue par la culture bourgeoise ${ }^{21}$. L'«art primitif» signifiait «le rejet de la tradition artistique capitalisée ». L'art moderne dénoncé par Einstein comme une « fiction formelle », producteur de marchandises au service de la spéculation et du snobisme, avait pour fin de «rassurer et conforter dans son for intérieur le bourgeois possédant». «Toute œuvre d'art est une œuvre de snobisme réactionnaire [...] si elle n'est pas subordonnée à la transformation sociale qui lui confère seule un sens. » Ce qu'il fallait, ajoutait Einstein, c'était un «art collectif : seule la révolution sociale implique la possibilité d'une transformation de l'art, elle en est la prémisse et elle seule détermine la valeur d'un changement artistique, elle seule confère à l'artiste sa tâche. [...] Si nous faisons exploser l'idéologie du capitalisme, nous trouverons, dans ses décombres, le seul vestige valable de ce continent défoncé, la condition préalable de toute nouveauté, la masse simple qui, aujourd'hui, est encore en proie à la souffrance. L'artiste, c'est elle ${ }^{22}$.» En quoi cette «masse simple » était-elle susceptible d'œuvrer pour la «transformation sociale »? Einstein ne le dit pas, mais rien, dans ses textes, ne suggère l'idée que cette contribution devait prendre la forme de sujets politiquement engagés ${ }^{23}$. Il est probable que le changement social radical était censé être la conséquence de cette production «collective», de son existence comme art fait par et pour le prolétariat. L'art africain, qui était alors au centre des préoccupations d'Einstein, était sans doute le modèle implicite de cet « art primitif »- non d'un point de vue formel, mais en tant qu'art émergeant du flux de la vie collective et intégré dans celle-ci. Nous reviendrons plus loin sur ce point.

Les espoirs placés par Einstein dans la révolution étaient partagés par beaucoup d'artistes et d'intellectuels dans l'atmosphère fébrile des années 1918-1919: un ordre social radicalement différent semblait alors possible, et des slogans en appelant à une intégration de l'art dans la vie des masses se faisaient partout entendre ${ }^{24}$. Cet enthousiasme semble avoir été sans lendemain, aussi bien pour Einstein que pour la majeure partie de l'intelligentsia de gauche. À partir de 1920, en tout cas, et pour plus d'une décennie, on ne trouve plus dans ses textes d'expression d'une solidarité avec la classe ouvrière ni d'une sympathie ouverte pour la politique de gauche. Les lettres qu'il adressa au cours de cette période à DanielHenry Kahnweiler et à Moïse Kisling suggèrent que, un an après avoir écrit «Sur l'art primitif ", Einstein fourmillait de nouveaux projets d'écriture et s'était pragmatiquement accommodé du monde artistique capitaliste ${ }^{25}$.

Que s'était-il passé? Aucun des écrits de cette époque, y compris les lettres qui nous sont parvenues, ne donne d'indice. Seuls les fragments déjà mentionnés de Bebuquin II (1934), qui se rapportaient à l'alter ego d'Einstein, fournissent quelques indications. Dans la révolution, écrivait-il, «BEB découvre chez les COMM[unistes] une attache : [...] un savoir naît de la vie commune" (souligné par Einstein). Là encore, le conflit demeurait profond car il craignait « une mécanisation de sa personne et de sa vie, une normalisation désespérée" menaçant de «détruire tous ses acquis personnels extraordinaires ${ }^{26}$ ». Incapable de noyer son individualité dans un mouvement de masse, «il cherche sans cesse à fuir les communistes... Le vieil anarchiste ne supporte pas les ordres, les slogans; ses critiques sans fin, son opposition ne cessent de transparaître. " Sa personnalité était « déjà à tel point artificiellement dressée et isolée qu'il ne pouvait plus s'adapter au COMM[unisme] ». Il manquait, se rendait-il compte, de "sentiment communautaire, par-delà les grandes phrases ${ }^{27}$ ».

\section{- $\bullet$}

21. Reproduit in Carl Einstein, Werke, t. II, op. cit., p. 27. Pour un commentaire plus développé de ce texte, voir Charles W. Haxthausen, «Bloody Serious: Two Texts by Carl Einstein », October 105, 2003, p. 112-115.

22. Carl Einstein, Werke, t. II, op. cit., p. 27 ; traduction infra, p. 185.

23. On peut penser, par exemple, à sa critique d'Otto Dix : «\| ose le kitsch qui objective notre temps, mais la peinture peut alors facilement tomber elle-même dans le banal; on fait trop confiance au motif excitant... Peut-être est-on, en peinture, un réactionnaire de cœur qui choisit des motifs de gauche »; Carl Einstein, Die Kunst des 20. Jahrhunderts, Berlin, Propyläen Verlag, 1926, p. 156, 157.

24. Voir Manfred Schlösser (éd.), Arbeitsrat für Kunst Berlin, 1918-1921: Ausstellung mit Dokumentation, Berlin, Akademie der Künste, 1980; Joan Weinstein, The End of Expressionism: Art and the November Revolution in Germany, 1918-19, Chicago, University of Chicago Press, 1990.

25. Voir les « Lettres de Carl Einstein à Moïse Kisling (19201924] », Liliane Meffre (éd.), Les Cahiers du musée national d'Art moderne 62, 1997, p. 74-123 ; Carl Einstein, Daniel-Henry Kahnweiler. Correspondance 1921-1939, Liliane Meffre (éd.), Marseille, André Dimanche Éditeur, 1993.

26. Carl Einstein, « Bebuquin II CEA, microforme 46, p. 150/104, daté du 22 janvier 1934.

27. Ibid., p. 188/132. Pour un examen détaillé de ce texte, voir Marianne Kröger, $B E B$ II, art. cit., p. 316-330. 


\section{CARL EINSTEIN ET LES PRIMITIVISMES}

\section{$\bullet \bullet$}

28. Ce texte est reproduit sous le titre «Revolution durchbricht Geschichte und Überlieferung » in Carl Einstein, Werke, t. IV [«Texte Aus dem Nachla $\beta$ | $)$ ], Hermann Haarmann et Klaus Siebenhaar (éd.), Berlin, Fannei \& Walz, 1992, p. 146-152; trad. : «La révolution brise l'histoire et la tradition », Cahiers du musée national d'Art moderne 117, 2011. Sur la datation de ce manuscrit, voir mon introduction à la traduction anglaise, « Revolution Smashes Through History and Tradition », October 107, 2004, p. 139-140.

29. Cf. Carl Einstein, «Dr. Breitscheid und das Rätesystem » (publié le 23 avril 1919 dans Die Rätezeitung), in id., Werke, t. II, op. cit., p. 25, 26.

30. Id., « Revolution durchbricht Geschichte und Überlieferung », Werke, t. IV, op. cit., p. 146-152.

31. Id., Die Kunst des 20. Jahrhunderts, op. cit., p. 82.

32. Einstein-Kahnweiler, Correspondance, op. cit., p. 48, 51, 139, 142.

33. Carl Einstein, Die Kunst des 20. Jahrhunderts, op. cit., p. 82.
Deux textes du début des années 1920, qui ne furent pas publiés sur le moment, attestent qu'Einstein n'avait pas perdu sa confiance dans la capacité de l'art à transformer fondamentalement la perception humaine et à renouveler le monde visuel. Le premier est un essai sans titre consacré à l'avant-garde soviétique, probablement écrit en $1921^{28}$. Une première lecture peut faire penser à une continuité avec la ligne de pensée de "Sur l'art primitif » : Einstein avait-il trouvé en Russie soviétique un art effectivement engagé dans la "transformation sociale", un «art collectif » œuvrant pour l'avènement d'une collectivité sociale? Il ne dit rien de l'agit-prop, ni du rôle des artistes dans la réorganisation des moyens de production, ni d'une quelconque autre fonction explicitement politique de l'art. Deux ans auparavant, confronté à la situation politique encore explosive de l'Allemagne, il se décrivait comme "communiste " et avait passionnément affirmé la viabilité d'une dictature du prolétariat ${ }^{29}$. À présent, dans ce texte consacré à la révolution russe et à son succès, il célébrait encore une dictature : non pas celle du prolétariat (qui n'était jamais évoqué!) mais celle de la vision (Diktatur des Sehens), la "dictature de l'homme contre les objets figés " - une vision dynamique, fonctionnelle, qui n'était pas la perception passive d'un monde d'objets stables mais un acte phénoménologique créatif, la figuration d'un nouvel ordre visuel. Le renoncement au moi n'était pas le produit des «liens avec les camarades de la masse", comme il l'avait affirmé en 1919, mais la conséquence d'un acte de vision non médiatisé, non entravé par la mémoire. «Dans le communisme, notait-il, la disparition du moi et la destruction de l'objet ne font qu'un ${ }^{30}$. » Mais il n'en disait pas davantage au sujet du communisme. Le problème envisagé n'était plus le même qu'en 1919 : ce n'était plus la contribution de l'art à une "transformation sociale " mais l'apport éventuel de la révolution à un projet de reconfiguration de la vision humaine. Les priorités d'Einstein se dévoilent dans le verdict négatif qu'il porte par la suite sur l'avant-garde soviétique, dont les œuvres contenaient selon lui «davantage de convictions politiques que de peinture; davantage de marxisme que quoi que ce soit d'autre ${ }^{31}$ ».

La notion de "disparition du moi» (Ichverschwindung) se retrouve, détachée cette fois de tout contexte politique, dans la longue lettre qu'Einstein adressa en juin 1923 à Kahnweiler. Il y était question du cubisme. Einstein voyait dans la peinture cubiste des potentialités de "transformation de la vision", mais aussi de "transformation de son équivalent linguistique et des sensations». Comme dans l'article sur la Russie, il décrivait cette vision active comme associée à un oubli temporaire du moi : "Cette sensation fait pour un certain laps de temps disparaître votre $\mathrm{Moi}^{32} »$ - mais il n'est pas question ici de subordination du moi au collectif.

Dans son premier long texte consacré au cubisme, L'Art du $x x^{e}$ siècle, Einstein revint pour la première fois depuis 1919 sur la question d'un art collectif. L'œuvre de Fernand Léger avait eu, de son point de vue, la particularité de donner naissance à une telle forme collective par la stylisation d'un "vécu collectif del'objet ». La géométrie des formes de Léger leur conférait une "signification collective »; "la dimension optique qui est commune aux choses est leur géométrie». «Le nombre est une marque de la collectivité urbaine (usine, armée, fabrication en série, parti), l'isolement est proscrit, l'accent est mis sur le communautaire ${ }^{33}$." Cet art collectif se fondait avant tout sur la communauté d'expérience visuelle dans la société industrielle capitaliste moderne - c'était un «art collectif » sans dimension politique collective.

Certains commentateurs ont vu là une appréciation positive de Léger, et il est vrai que tous les textes où Einstein évoque ce dernier sont empreints de sympathie 


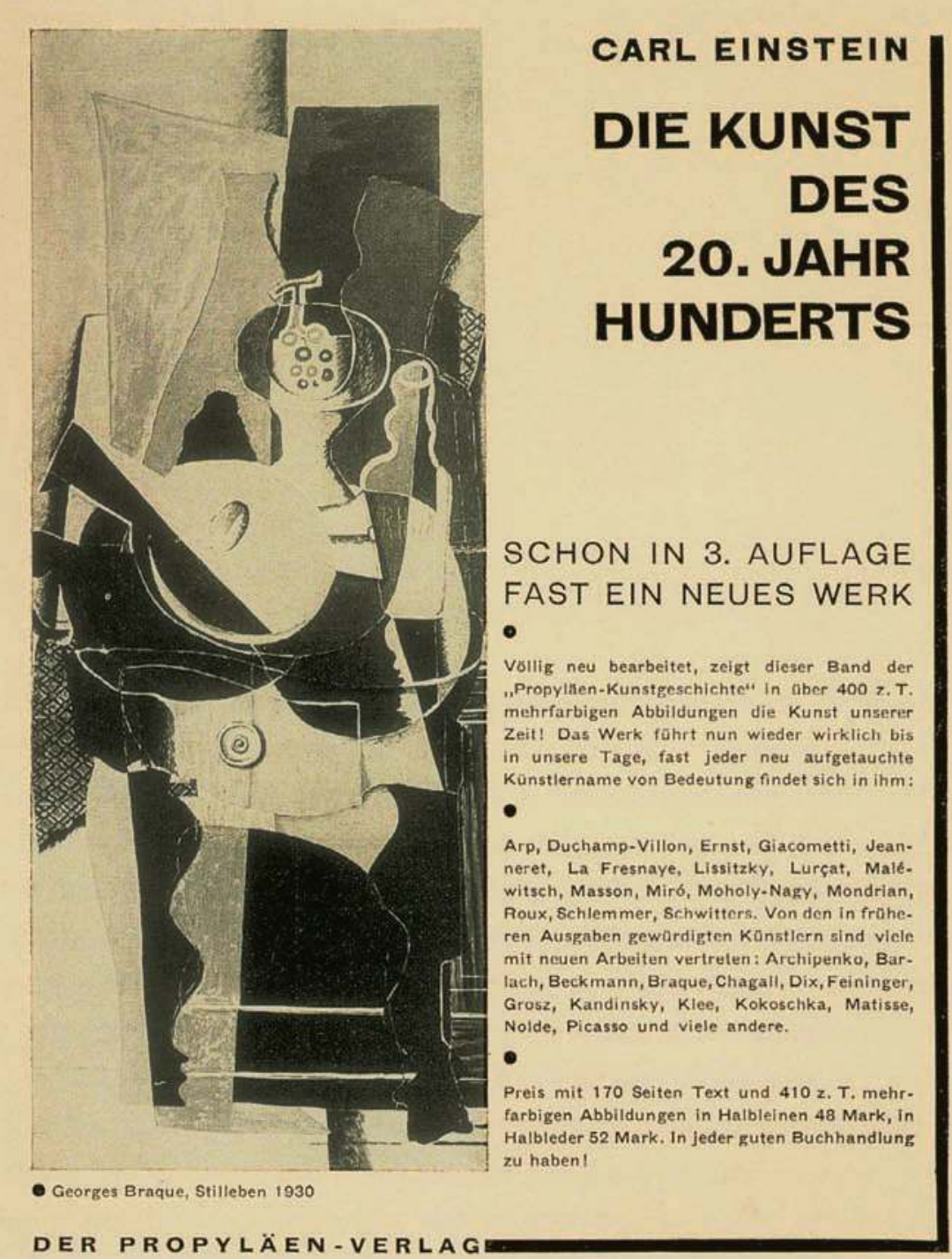

191

Fig. 5 Annonce pour la troisième édition de Die Kunst des 20. Jahrhunderts avec Nature morte de Georges Braque [1930], 1932.

et de respect; plus tard, il désignera tout simplement le peintre comme " un homme de la collectivitée ${ }^{34}$ ». Pourtant, à y regarder de plus près, il est clair que, en stylisant le monde des objets technologiques modernes et en leur prêtant une signification collective, Léger n'était pas engagé dans la «destruction de l'objet» réclamée par Einstein. Ses tableaux étaient «trop marqués par un monde de formes et de finalités étrangères; on n'échappe pas totalement au descriptif, on ne fait que l'appliquer à des formes déjà construites 35 ». Le rationalisme du peintre, sa "négation du fait psychologique et de l'individu, écrira-t-il plus tard, expliquent pourquoi l'art de Léger est apprécié par les Russes soviétiques, par les Américains, et par certains groupes à l'esprit très industriel ${ }^{36} "$. Rien, dans les écrits d'Einstein, ne peut faire penser que cette remarque ait été conçue comme un éloge.

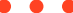

34. Id., «Léger : œuvres récentes 》 (1930), in Ethnologie d'art moderne, Liliane Meffre (éd.), Marseille, André Dimanche Éditeur, 1993, p. 48. Sur Einstein et Léger, voir Klaus H. Kiefer, «Diskurswandel... », art. cit., p. 362-364. Léger lui-même avait écrit en 1913 : « Nous arrivons, j'en suis persuadé, à une conception de l'art aussi vaste que les plus grandes époques précédentes: même tendance aux grandes dimensions, même effort partagé par une collectivité. » Fernand Léger, « Les origines de la peinture et sa valeur représentative » (1913), repris in id., Fonctions de la peinture, Paris, Gonthier, 1965, p. 16.

35. Carl Einstein, Die Kunst des 20. Jahrhunderts, op. cit., p. 80. Ailleurs, Einstein critique le privilège selon lui accordé par Léger au nombre, au quantifiable : «Le nombre est l'outil d'une pensée rétrospective. » [《Totalität », Werke, t. I, op. cit., p. 220).

36. Carl Einstein, Werke, t. III, op. cit., p. 521. 


\section{CARL EINSTEIN ET LES PRIMITIVISMES}

\section{- $\bullet$}

37. Id., Die Kunst des

20. Jahrhunderts, op. cit., p. 61.

38. Voir, par exemple, sa discussion sur Picasso dans L'Art du xx siècle, Berlin, Propyläen Verlag, 1928, p. 68, 69. Einstein évoquait «l'abandon de la personne »: «Qu'est-ce qui marque le plus l'unité de la personne sinon, dans le meilleur des cas, la possibilité d'être éclaté à travers la multiplicité des visions; l'âme vit d'une vie plus intense dans ce changement explosif que dans l'identité étroite des répétitions. »

39. Cf. Rolf-Peter Baake (éd.), Carl Einstein. Materialien, I: Zwischen Bebuquin und Negerplastik, Berlin, Silver \& Goldstein, 1990, p. 138. Baake date la lettre de 1916.

40. Carl Einstein, Afrikanische Plastik, Berlin, Verlag Ernst Wasmuth, 1921.

41. Id. (éd.), Afrikanische Legenden, Berlin, Rowohlt, 1925.

42. Emil Torday et Thomas Athol Joyce, Notes ethnographiques sur les peuples communément appelés Bakuba, ainsi que sur les peuplades apparentées, les Bushongo, Bruxelles, ministère des Colonies, en vente chez Falk fils, 1910. La collaboration entre Einstein et Joyce est documentée dans les lettres du premier à Ewald Wasmuth, conservées au Deutsches-Literatur-Archiv de Marbach, et dans des lettres d'Einstein à Joyce conservées dans les archives du British Museum.

43. J'ai puisé une partie de ces informations dans l'article d'Heike Neumeister, « Masks and Shadow Souls: Carl Einstein's Collaboration with Thomas A. Joyce, The British Museum and Documents », in Nicola Creighton et Andreas Kramer (éd.), Carl Einstein and the European Avant-garde, à paraître.

44. Carl Einstein, « Das Berliner Völkerkunde-Museum: Anläßlich der Neuordnung » (1926), Werke, t. Il, op. cit., p. 446-450.
Dans la première édition de L'Art du $x x^{e}$ siècle, Einstein ne créditait pas encore Picasso et Braque de la réalisation d'une forme collective, mais il offrait une vision alternative des modalités d'existence d'une telle forme. La vision subjective individuelle, "dans laquelle s'exprime ce qui est spécifiquement humain - appelons-le le poétique", induisait une forme de "validité qui incite à la formation de collectifs, de groupes ${ }^{37}$ ». Avec les formes désindividualisées de Léger, l'acte de perception ne s'accompagnait pas d'une transformation du sujet qui voit. Or, pour Einstein, la «suprématie de l'acte subjectif » était primordiale. "C'est en lui, en tant qu'acte créatif, que réside la composante décisive de l'acte de vision, et c'est ainsi qu'on évite la prédétermination par le donné " (c'est l'auteur qui souligne), à laquelle la peinture de Léger n'échappait pas. Quelques années plus tard, Einstein revint à la notion de "disparition du moi ", une conception du processus artistique dans laquelle le sujet identique à lui-même, artiste ou spectateur, était momentanément dissous, comme possédé par un esprit ${ }^{38}$. En d'autres termes, il transposa au cas de l'art moderne la notion animiste d'agentivité décrite dans Negerplastik.

Negerplastik fut le premier fruit de l'investissement d'Einstein dans l'étude de l'Afrique et de son art. C'est peut-être la renommée de l'ouvrage qui lui valut d'obtenir en 1916, durant son service dans la Belgique occupée, une place au ministère des Colonies à Bruxelles. Einstein eut ainsi accès aux importantes collections africaines et à la bibliothèque du musée du Congo de Tervuren. Comme il l'écrivit avec enthousiasme à Franz Blei : «Ici, je négrifie complètement. Un excès africain. » Il avait le projet d'écrire deux nouveaux livres sur l'Afrique ${ }^{39}$. Un an après la publication de la seconde édition de Negerplastik (1920), Einstein publia La Sculpture africaine (Afrikanische Plasti $k^{40}$ ). Ce mince volume offrait une analyse de la sculpture africaine plus concrète que le texte fameux qui l'avait précédé - plus académique que théorique, plus ethnographique que stylistique. Einstein rapportait les objets aux mythes et aux rituels africains, il avait inclus cette fois les mises en contexte qui faisaient défaut dans le premier livre. À cette date, aucun autre historien de l'art ou critique d'art n'avait écrit un texte aussi détaillé sur la sculpture africaine. En 1925, il publia Légendes africaines (Afrikanische Legenden), une collection de transpositions de contes et de mythes africains, auxquelles était adjointe une longue bibliographie d'ouvrages ethnologiques ${ }^{41}$. La même année, il séjourna pendant une assez longue période à Londres, où il collabora avec Thomas Athol Joyce, responsable des collections ethnographiques du British Museum, lui-même coauteur d'une importante étude sur les Bakuba ${ }^{42}$. Ensemble, ils travaillèrent à un projet de livre : une étude historique compréhensive des arts des peuples africains. Puis Joyce fut pris par d'autres tâches et, en dépit de nombreux efforts de part et d'autre pour relancer le projet, celui-ci ne fut jamais mené à son terme ${ }^{43}$. Cet investissement approfondi dans l'étude de l'Afrique et de son art devait avoir un impact puissant sur Einstein et l'évolution de ses idées concernant l'agentivité de l'art.

Même dans les années 1920, où Einstein consacra l'essentiel de ses écrits à l'art moderne, le modèle d'un art intégré dans les pratiques de la vie, dont les cultures primitives africaines représentaient pour lui l'exemple par excellence, semblait toujours présent à son esprit. C'est ce qui ressort d'un texte publié en 1926 au sujet de la réorganisation du musée d'Ethnologie de Berlin ${ }^{44}$. Dans la première partie, Einstein déplore la perte de la fonctionnalité de l'art, la rupture avec un temps où celui-ci était intégré dans des systèmes de croyance collectifs, un modèle de 


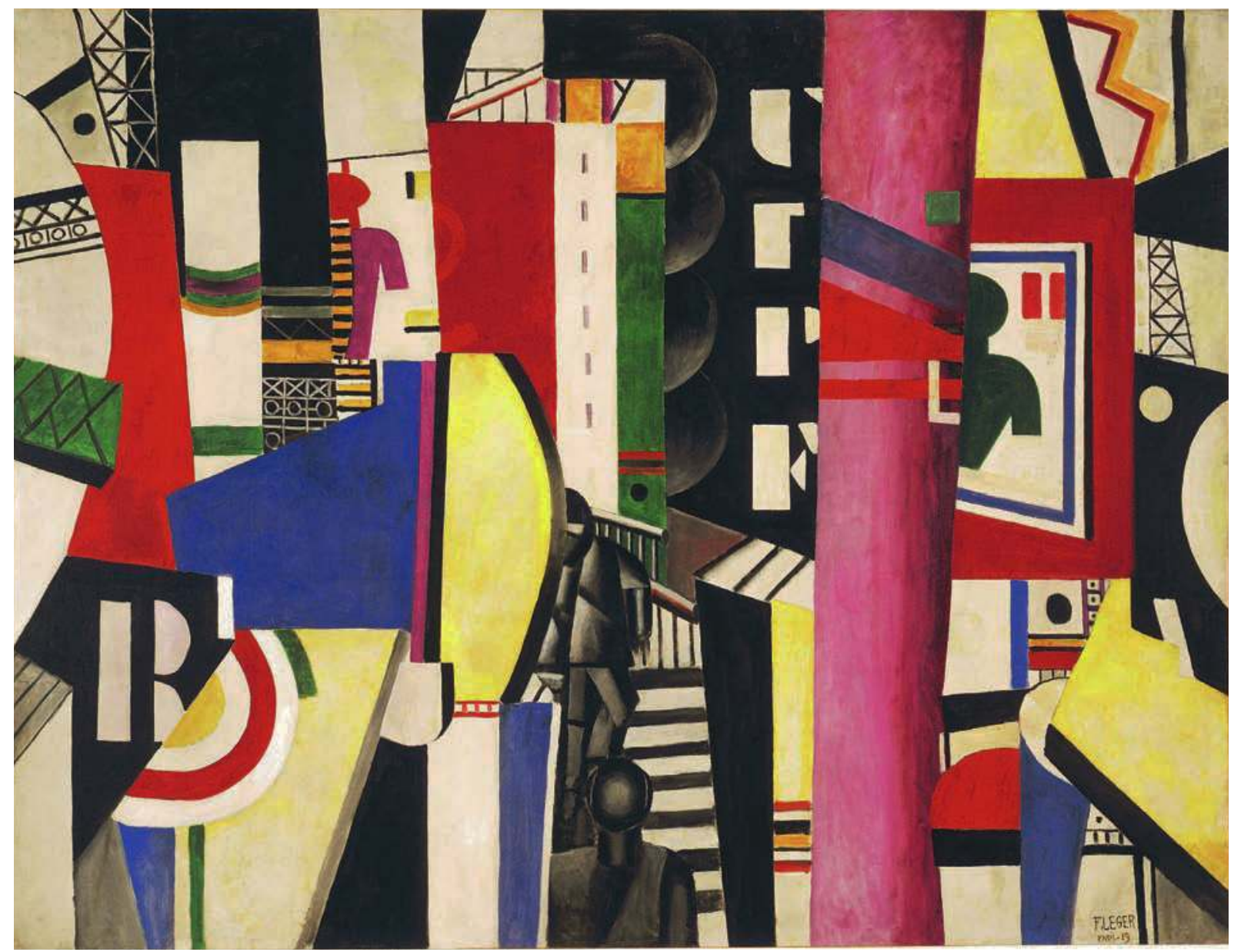

Fig. 6 Fernand Léger, La Ville, 1919, reproduit dans Die Kunst des 20. Jahrhunderts. The Philadelphia Museum of Art / Art Resource / Scala, Florence @ ADAGP, Paris 2011. 


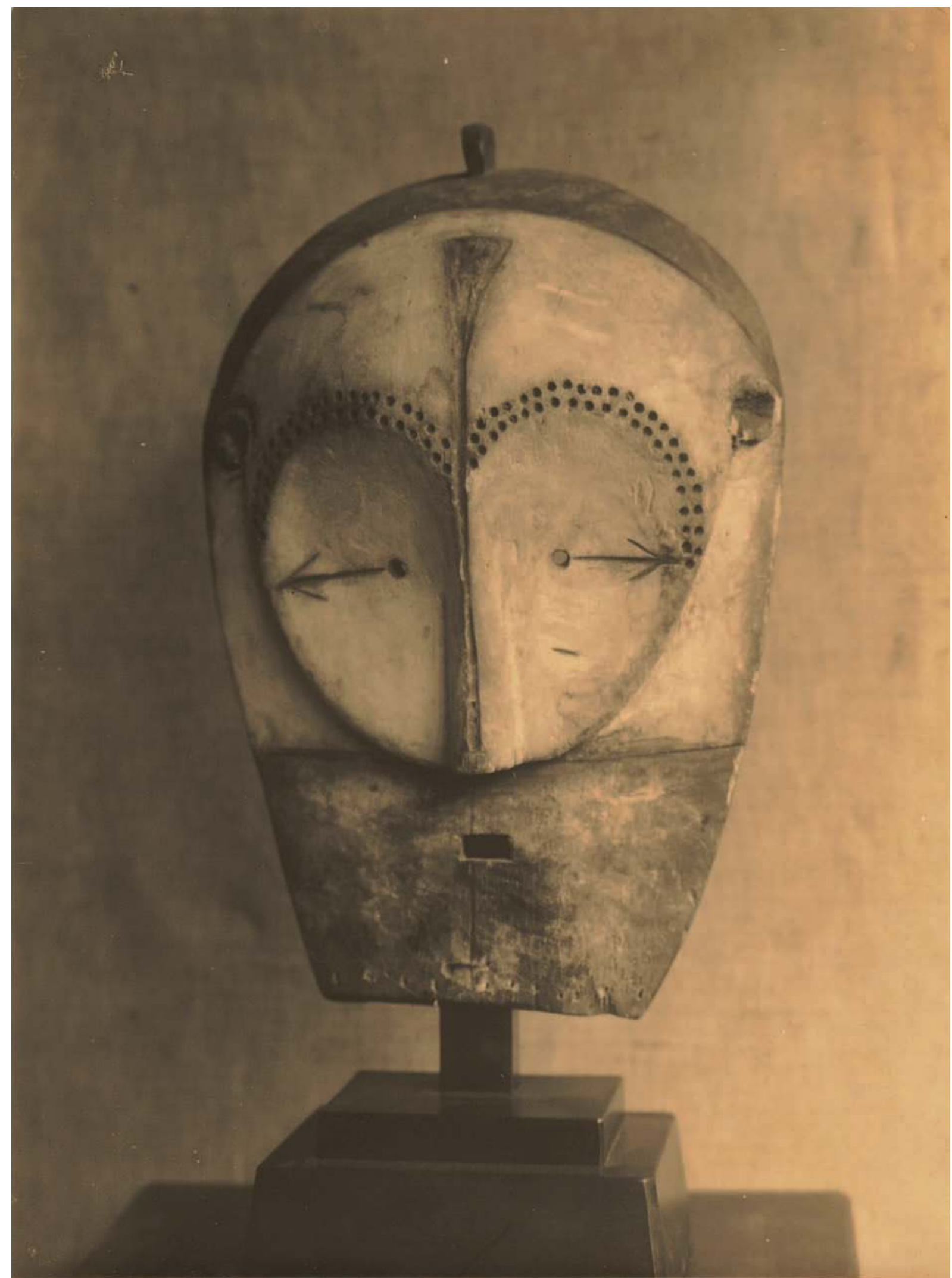

Fig. 7 Man Ray, Masque fang, s.d. Collection particulière, Paris @ MAN RAY TRUST, ADAGP, Paris 2011. 
fonctionnalité inimaginable dans la société contemporaine sécularisée. «Dès lors qu'on se mit à considérer que chacun des arts valait par lui-même, tout le caractère de ces derniers s'en trouva modifié. Ils furent coupés de l'au-delà de la foi vivante et étudiés du point de vue de leur valeur formelle." "Le retable était beau parce qu'il était ceint des angoisses, des désirs et des cris de terreur qui montaient vers Dieu, parce qu'il était la partie la plus modeste de toute une action, parce qu'il était habité par l'ombre du Dieu et servi par des prêtres, non des employés de musée ${ }^{45}$.»

En 1929, après son départ de Berlin et son installation à Paris, Einstein crut semble-t-il avoir découvert une pratique artistique dans laquelle l'art moderne avait retrouvé une part de son agentivité perdue. Cet art était le surréalisme. Au début des années 1930, sous l'influence de ce qu'on a appelé le «surréalisme ethnographique ${ }^{46}$ ", Einstein pensa que le fossé entre l'art de l'avant-garde européenne qu'il défendait et les pratiques artistiques des cultures primitives et archaïques était désormais comblé; l'art pouvait jouer un rôle dans le processus auquel il donnait le nom de "primitivisation », pour désigner un phénomène de retour à une vision du monde collective et mythique. On le voit bien dans les réflexions sur l'art contemporain qu'il développa parallèlement à son essai de 1930 sur la grande exposition d'objets africains et océaniens présentée à la galerie du Théâtre Pigalle ${ }^{47}$. Désormais, à la différence de ce qui s'était passé pour Negerplastik, Einstein ne formulait que peu de remarques sur la forme; il se focalisait avant tout sur les fonctions rituelles des objets. Il insistait davantage que par le passé sur le caractère animiste de la sculpture africaine. C'était là un «dogme fondamental » : "Tout esprit peut habiter dans tout objet. » "La chose décisive est moins la statue que le fait qu'elle soit habitée par un esprit, et la ressemblance avec l'esprit ne consiste pas dans une ressemblance individuelle, mais dans la représentation des marques magiques et collectives ${ }^{4}$. " Fait significatif, Einstein avait publié en 1929 dans Documents un article sur André Masson au sous-titre provocateur, «Étude ethnologique». De la même façon qu'il décrivait comment, dans les cultures africaines, l'itongo, ou l'âme, pouvait habiter "des pierres, animaux ou plantes 49 ", il désignait l'art de Masson comme un "training extatique » dans lequel le "moi éclipsé " se soumettait à des «identifications remarquables [...] avec les plantes, les étoiles, les pierres ${ }^{50} \%$.

Au demeurant, pour Einstein, c'était moins l'art des surréalistes proprement dits qui incarnait le mieux cet idéal que celui de Picasso, Braque et Paul Klee ${ }^{51}$. Aucun texte ne le fait apparaître plus clairement que son dernier grand livre sur l'art moderne, Georges Braque ${ }^{52}$. Dans ce texte, il attribue aux peintures récentes de Braque et, implicitement, à celles de Picasso, l'agentivité d'esprits habitant l'objet, au sens animiste caractéristique des cultures primitives. L'œuvre d'art naissait alors de ce qu'Einstein appelait un «intervalle hallucinatoire» dans le processus de création artistique, qui impliquait un oubli de soi temporaire et devait plonger le spectateur dans un état halluciné. Dans ce processus, l'œuvre d'art remplissait la même fonction "que les idoles anciennes; par là, on se trompe soi-même et on croit que les œuvres d'art, comme les esprits des morts, possèdent le pouvoir de revenir en restant les mêmes. Voilà pourquoi on craint secrètement les images, comme le primitif » : «elles vivent et meurent comme si elles étaient réelles et elles font l'effet d'être étrangement vivantes ${ }^{53}$ ». C'est par le biais d'une telle expérience, à l'évidence, qu'Einstein croyait à la possibilité d'une réalisation de "l'utopie révolutionnaire " par l'art. Il est bien difficile de préciser ce qu'il entendait par là, car il n’a jamais approfondi cette idée. Durant cette

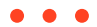

46. J'emprunte l'expression à James Clifford, « On Ethnographic Surrealism », in The Predicament of Culture, Cambridge (Mass.), Harvard University Press, 1988, p. 117-151.

47. Carl Einstein, «À propos de l'exposition de la galerie du Théâtre Pigalle » (1930), Ethnologie d'art moderne, op. cit., p. 67-78.

48. Ibid., p. 72, 73.

49. Ibid., p. 71, 72.

50. Carl Einstein, «André Masson : étude ethnologique », in Ethnologie d'art moderne, op. cit., p. 25.

51. Voir Charles W. Haxthausen, «"Die erheblichste Persönlichkeit unter den deutschen Künstlern" : Einstein über Klee », in Die visuelle Wende der Moderne. Carl Einsteins "Die Kunst des 20. Jahrhunderts", Klaus H. Kiefer (éd.), Munich, Wilhelm Fink Verlag, 2003, p. 131146.

52. Carl Einstein, Georges Braque, op. cit., p. 65 (traduction modifiée par Isabelle Kalinowski].

53. Ibid., p. 52. 
0

54. Carl Einstein, Die Kunst des 20. Jahrhunderts [1931], Uwe Fleckner et Thomas W. Gaethgens (éd.), Berlin, Fannei \& Walz, 1996, p. 116, 122

55. Ibid., p. 116.

56. Ibid., p. 119.

57. Carl Einstein, « Pablo Picasso : quelques tableaux de 1928 » [1929], in Werke, t. III, op. cit., p. 17-24, ici p. 19.

58. Carl Einstein, Georges Braque, op. cit., p. 52 (traduction modifiée par Isabelle Kalinowski).

59. Ibid., p. 53 période, "l'utopie révolutionnaire" ne semblait consister que dans une culture "collective", mythique, dont les contours n'étaient pas plus précisément définis. Einstein semblait croire qu'une œuvre d'art créée pour une part dans un état provisoire d'hallucination était à même d'atteindre cet objectif. Paradoxalement, il fallait passer par une expérience subjective de l'artiste individuel, analogue à la transe, pour qu'un monde visuel collectif puisse s'imposer aux spectateurs de l'œuvre d'art 54 .

Dans ce processus de mise au jour d'un monde visuel collectif, un facteur jouait un rôle central, à savoir ce qu'Einstein appelait le "tectonique", concept capital pour lui dès ses premiers textes de critique d'art. À l'origine, le tectonique était une qualité, un principe d'organisation à l'œuvre dans des images. En 1931, au demeurant, le sens de la notion s'était élargi, chez Einstein, à une dimension psycho-anthropologique, anachronique. Les formes tectoniques étaient un rempart contre les contingences de la vie organique et, en dernière instance, contre la mort; elles étaient identifiées à des symboles sexuels archaïques, "des signes collectifs », comme Einstein les appelait, signes de la force vitale et - c'était là l'aspect essentiel - le tectonique était enraciné dans les strates les plus profondes de la mémoire collective, dans la «couche inférieure de l'inconscient, archaïque et collective ${ }^{55}$ ». C'est à travers ces formes tectoniques que l'artiste était appelé à créer des "objets nouveaux ${ }^{56}$ ", à générer les "créatures d'une mythologie des formes " dotée d'une validité collective ${ }^{57}$.

Il est remarquable qu'Einstein ait décrit ce processus en des termes très voisins de ceux qu'il employa, deux ans plus tard, pour relater son expérience de la révolution de 1918-1919. Ce qui n'avait pu être obtenu par un acte de volonté politique, à savoir la dissolution du moi dans une collectivité, pouvait désormais, affirmaitil, être réalisé par la transe hallucinatoire involontaire induite par l'œuvre d'art, à même de provoquer une dissolution momentanée de l'ego et une transformation du sujet de la vision. Einstein décrivait ce processus de façon très détaillée dans le cinquième chapitre de Georges Braque :

C'est justement ce processus de devenir-inconscient qui livre le spectateur d'autant plus brutalement à l'action de l'œuvre d'art: celle-ci acquiert sur la personne du spectateur rendu à son inconscient archaïque le pouvoir supérieur d'un démon ou d'un esprit. [...] Dans cette fixation des forces psychiques sur l'œuvre d'art, le moi et la conscience du temps s'effacent. Le moi disparaît car l'énergie du spectateur est projetée dans l'image, de la même manière que l'orant transpose son énergie dans le dieu qu'il vénère ${ }^{58}$.

Les phénomènes évoqués ici rappellent les descriptions de Negerplastik, mais ils concernent à présent l'œuvre d'art moderne, profane. C'est l'identification avec l'œuvre d'art, et non plus avec le dieu, qui induit l'oubli de soi, et cette " anesthésie " offre « une chance de liberté humaine » par «la mise au jour de la strate la plus profonde de la personnalité, la personne hallucinatoire ${ }^{59}$ ".

Mais comment la «collectivité » produite par l'expérience de l'œuvre d'art pouvait-elle se traduire dans des structures sociales et politiques? Einstein, de fait, n'en dit mot. Au début des années 1930, son édifice théorique utopique complexe présentait des failles. Einstein finit par reconnaître l'isolement social de l'avant-garde; l'art était pratiqué et consommé par une élite coupée du reste de la société. Cette thématique, articulée dans l'essai de 1919, "Sur l'art primitif", se retrouve dans le chapitre de L'Art du xx siècle consacré au surréalisme, puis dans Georges Braque. Einstein décrivait le prolétariat industriel comme «une masse qui ne connaissait pas grand-chose à la culture existante; ce qui posait 


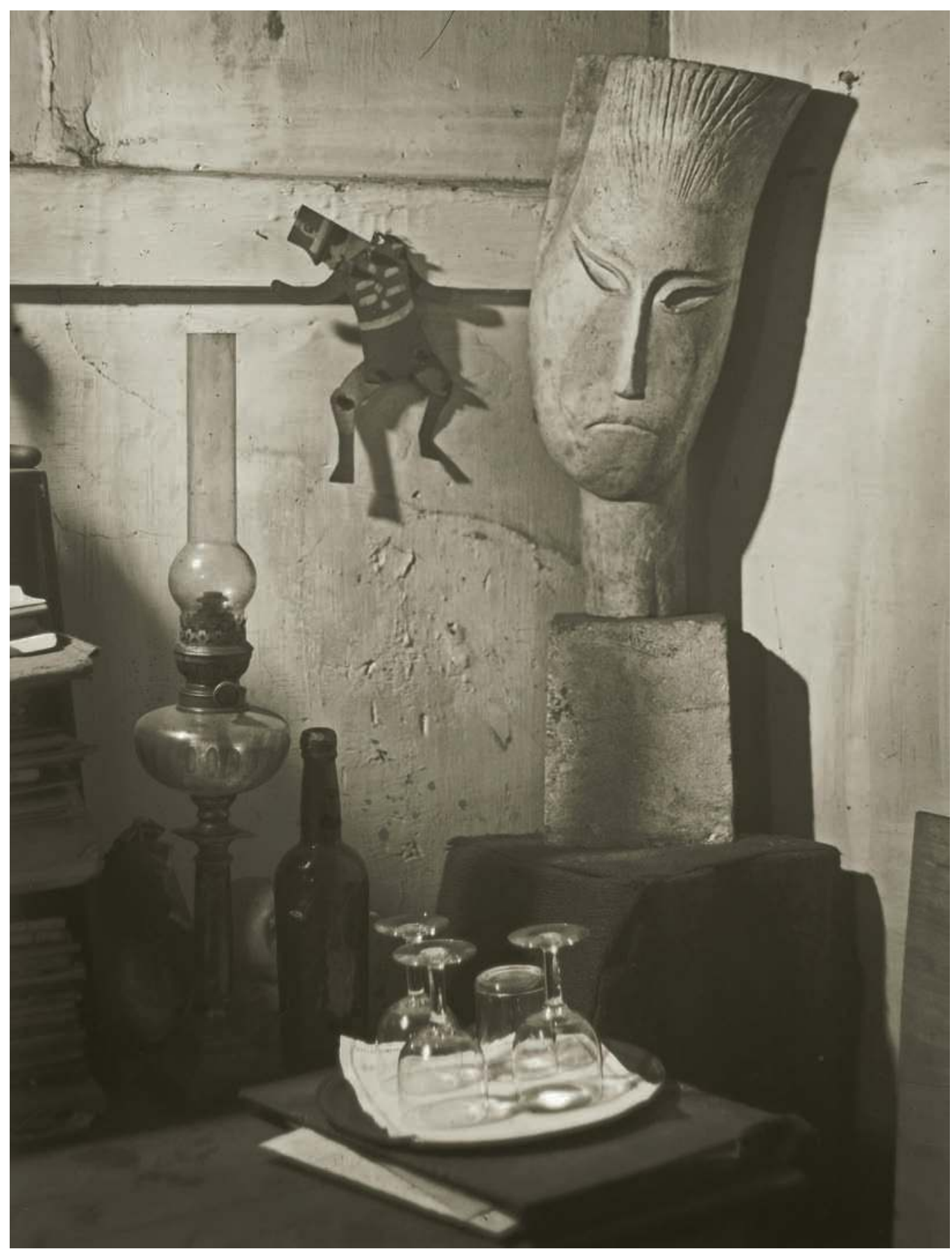

Fig. 8 André Kertész, Chez Ossip Zadkine, à Paris, 1926, négatif de l'auteur, négatif monochrome @ ministère de la Culture - médiathèque du Patrimoine, dist. RMN / André Kertész. 


\section{CARL EINSTEIN ET LES PRIMITIVISMES}

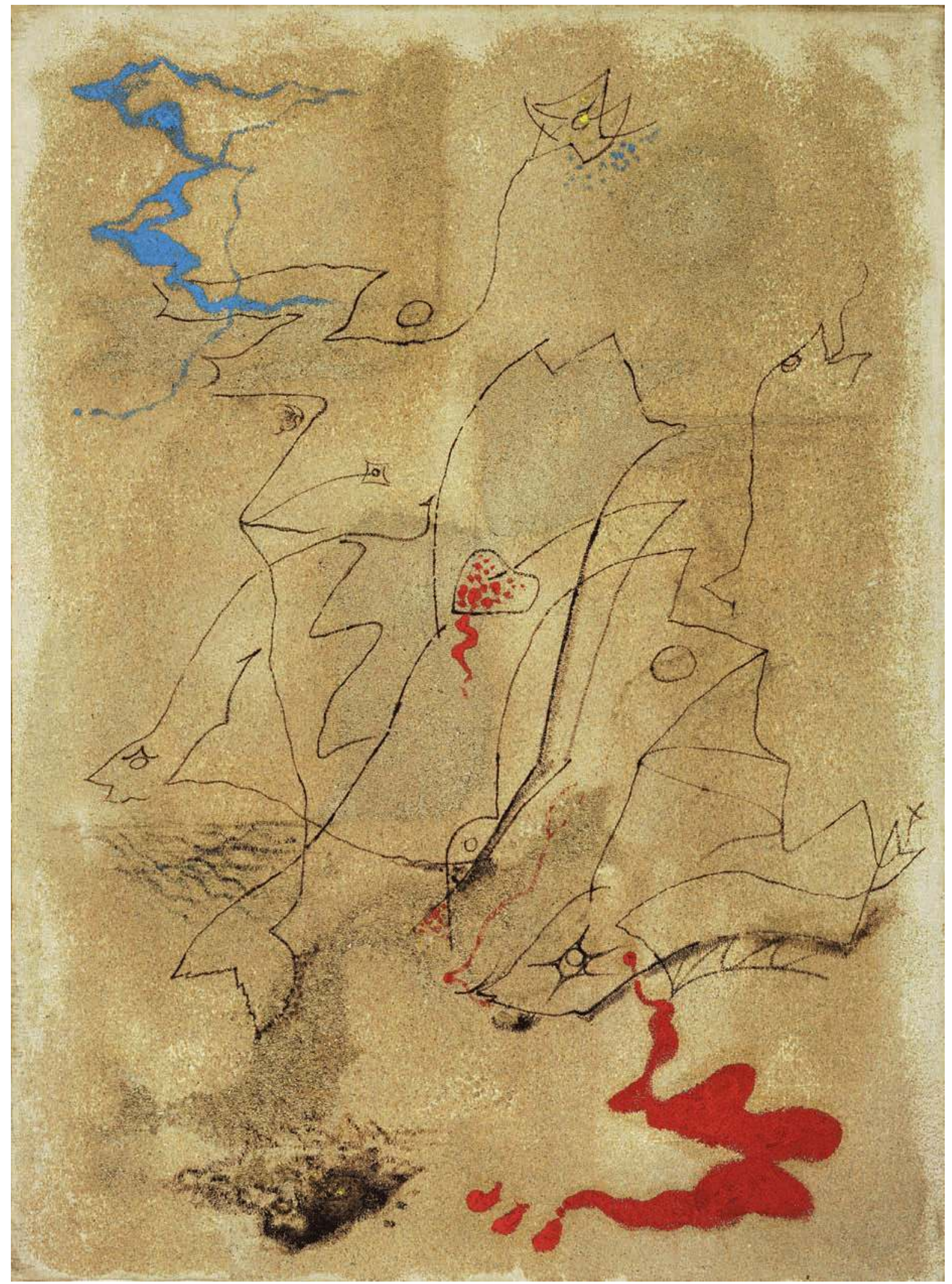

Fig. 9 André Masson, Les poissons dessinés sur le sable, 1927, reproduit dans «André Masson : étude ethnologique ", Documents vol. I, $n^{\circ} 2$, 1929, et dans Die Kunst des 20. Jahrhunderts. Kunstmuseum Berne, collection Rupf @ ADAGP, Paris 2011. 
le problème, encore irrésolu aujourd'hui, de savoir si cette culture était adaptée à une classe plus primitive ou si cette dernière devait fatalement être embourgeoisée. Les intellectuels et les artistes d'Europe occidentale n'ont guère su, jusqu'ici, adopter une position sur ce point et c'est ce qui explique leur isolement croissant ${ }^{60}$. " Einstein considérait que le prolétariat avait des besoins plus urgents que celui de l'art : il fallait s'habituer à l'idée «que, temporairement, des époques sans art étaient possibles et nécessaires ${ }^{61}{ }^{\prime}$.

De façon significative, c'est pendant le travail sur Georges Braque, où l'articulation de la théorie d'Einstein est la plus développée, que sa confiance dans ce projet critique s'effondra; ce dernier ne lui inspira plus, dès lors, que du dégoût. En septembre 1931, il annonça à son ami Ewald Wasmuth que son livre sur Braque serait le dernier : «J'en ai assez, je suis écœuré. Ras-le-bol aussi des théories. Cela fait trop de temps que ces tapisseries nous collent à la peau ${ }^{62}$. " Le monde de l'art était une "supercherie »; Einstein était «fatigué et dégoûté de tout. [...] Ou'ils continuent à faire tourner leur cirque sans moi. [...] J'en ai assez de cette industrie du génie, mercantile à un point si répugnant ${ }^{63}$. " La distance prise avec les idées exposées dans le Georges Braque, publié en 1934 avec un retard considérable, était perceptible dans la note du frontispice qui datait l'œuvre de 1931-1932. C'est à la même époque qu'Einstein rédigea la note citée au début de cet article, où il tournait lui-même en ridicule sa quête d'une « utopie révolutionnaire " qu'il espérait voir réalisée "par un changement de la forme artistique ».

Après l'achèvement du livre sur Braque, Einstein publia encore un essai substantiel, mais qui ne portait pas sur l'art moderne. Ce texte, traduit dans le présent numéro, était l'introduction au catalogue d'une exposition présentée dans une galerie privée de New York et regroupant des statuettes anciennes, hittites, étrusques, grecques et égyptiennes. C'est le seul texte publié par Einstein de son vivant dans lequel il articula la révision de son jugement sur l'art moderne. Se plaçant à une échelle historique plus vaste, il prenait acte du fossé béant qui séparait l'art moderne de ces cultures anciennes. «Les œuvres de la haute Antiquité étaient au service d'une image du monde magique et dotée d'une signification et elles étaient par là vouées à la conservation des dieux et du peuple [...]. Une statue représentait alors bien davantage qu'une imitation ou une création de formes isolée; elle était au service d'objectifs de première importance, à savoir la préservation et le renforcement de la vie ${ }^{64}$. " Le contraste avec la modernité était évident: "L'art s'est volatilisé dans un brouillard vague, et les œuvres s'enlisent dans le marais des paraphrases. [...] Avouons-le sans détours, l'art a incroyablement perdu de sa puissance spirituelle, parce qu'il n'est plus affecté au service d'un ordre puissant et strict. L'art seul n'est pas en mesure d'en créer un, et les critères exclusivement esthétiques ou formels traduisent précisément la pauvreté spirituelle d'une grande part des images actuelles ${ }^{65}$. " (C'est l'auteur qui souligne.) Ce bref passage marque, chez Einstein, une révision majeure de sa conception utopique de l'agentivité de l'art.

Einstein développa très longuement cette idée dans La Fabrication des fictions. Dans ce texte, il relativisait beaucoup la place de l'avant-garde dans l'ensemble de la société. Ce manuscrit inédit, probablement écrit à la fin de l'année 1935 ou au début de l'année 1936, se présentait comme une longue diatribe cathartique de 500 pages $^{66}$. Einstein s'y livrait à une critique impitoyable et corrosive du modernisme qui n'épargnait pas ses propres illusions passées. Il s'en prenait aux artistes et aux intellectuels, arrogants, élitistes et imbus d'euxmêmes, pseudo-révolutionnaires choyés qui s'employaient à distraire les classes

\section{- $\bullet$}

60. Carl Einstein, Die Kunst des 20. Jahrhunderts [1931], op. cit., p. 15 ?.

61. Id., Georges Braque, op. cit., p. 37 (traduction légèrement modifiée).

62. La lettre, écrite à la machine et postée de Paris, était datée «28.XV[sic].31 »; archives Ewald Wasmuth, Deutsches LiteraturArchiv, Marbach. Dans la même lettre, Einstein évoque la visite officielle du Président du Conseil français Pierre Laval à Berlin, qui eut lieu à la fin de septembre 1931; la lettre date sans doute à peu près du même moment.

63. Lettre à Ewald Wasmuth, non datée, probablement écrite durant l'été 1932.

64. Carl Einstein, Exhibition of Bronze Statuettes B.C. - Hittite, Etruscan, Egyptian, Greek (1933), in Werke, t. III, op. cit., p. 224; traduction française dans ce numéro, infra, p. 234.

65. Id., ibid.

66. Voir note 3. 
dominantes et à préserver leur propre statut privilégié. Il réfutait point par point les positions de ses textes antérieurs. En voici quelques passages représentatifs :

L'avant-garde a travaillé dans un état d'isolement anarchique. Elle a été incapable de construire et d'imposer une vision susceptible d'influencer une collectivité. [...]

Le crime des intellectuels a été de s'arroger tous les privilèges de la création, exactement comme les entrepreneurs capitalistes ont revendiqué le monopole de la production. Ces deux groupes se sont placés à l'écart des faits collectifs et de l'action de la communauté. Ils ont adopté une attitude aristocratique d'exclusivité. Ils ont regardé l'histoire et la masse comme un objet inepte et secondaire et se sont réservé tous les privilèges de la valeur et de l'ordre. [...]

Les styles importants sont nés dans des situations et des circonstances dont le caractère et la culture étaient déjà définis. À ce qu'il semble, les poètes ou les peintres ne sont pas capables de conférer à l'existence un sens primaire ni de forger une réalité primaire. L'art ne semble s'épanouir en une riche floraison que lorsque les problèmes élémentaires, tels que l'organisation sociale, les formes de vie, les visions, etc. sont déjà fixés et n'ont plus qu'à être confirmés dans des images. Les modernes, à la mentalité idéaliste, ont eu tort de croire qu'on pouvait donner naissance à une réalité contraignante par la voie de l'imagination, tout en restant isolé et coupé de la société. [...]

La modernité d'aujourd'hui passera, car ce qui lui fait défaut, ce sont des attaches et des normes sociales. Cet art périra par excès de formalisme autiste et de gratuité coupée du réel. [...]

Les peintres trouvèrent dans les formes tectoniques un collectif de substitution qui devait atténuer l'excès de formalisme subjectif. Ils étaient restés les mêmes artistes. La quête de formes contraignantes s'acheva dans l'erreur et la résignation. La tectonique d'images qui ne correspond pas à une société tectonique n'est qu'un sport musical ${ }^{67}$.

La dernière de ces citations soulevait une question qu'Einstein n'avait jusquelà jamais abordée de front : en quoi consistait le lien entre l'image tectonique et la société tectonique - c'est-à-dire le collectif? Dans La Fabrication des fictions, il concédait finalement qu'un tel pont n'existait pas. Il fallait donc trouver une autre voie, en sens inverse : "Un nouveau style spirituel ne pourra être possible qu'au terme d'une révolution qui créera une situation sociale nouvelle et des types humains différents. Les intellectuels doivent retrouver le sens du travail commun utile et abandonner l'utopie de l'action esthétiquement parfaite mais dépourvue de finalité. Les conventions artistiques ne sont que les conséquences secondaires de consensus sociaux déterminants. Ce sont ces derniers qui leur confèrent un sens ${ }^{68}$."

En 1936, Einstein vit dans les débuts de la guerre civile espagnole l'opportunité d'un tel "travail commun utile", une chance de renouveau et de rédemption de sa vie. Il partit pour Barcelone avec sa femme Lyda, quittant Paris, comme il le relata plus tard à Kahnweiler, "sans dire un mot ${ }^{69}$ ». En Espagne, il rejoignit la milice anarchosyndicaliste de Buenaventura Durruti, composée de "travailleurs, de prolétaires des usines et des villages ». Une remarque formulée dans l'allocution radiophonique, traduite dans ce numéro, qu'Einstein consacra en 1936 à son expérience au sein de la colonne Durruti fait le lien avec le Georges Braque et le fragment de Bebuquin II sur la révolution allemande, parus deux ans auparavant: "Durruti [...] avait banni de la grammaire le "moi", ce mot préhistorique. Dans la colonne Durruti, on ne connaît que la syntaxe collective. Les camarades vont apprendre aux littérateurs à renouveler la grammaire dans un sens collectif. [...] Durruti avait reconnu au plus profond de lui-même 


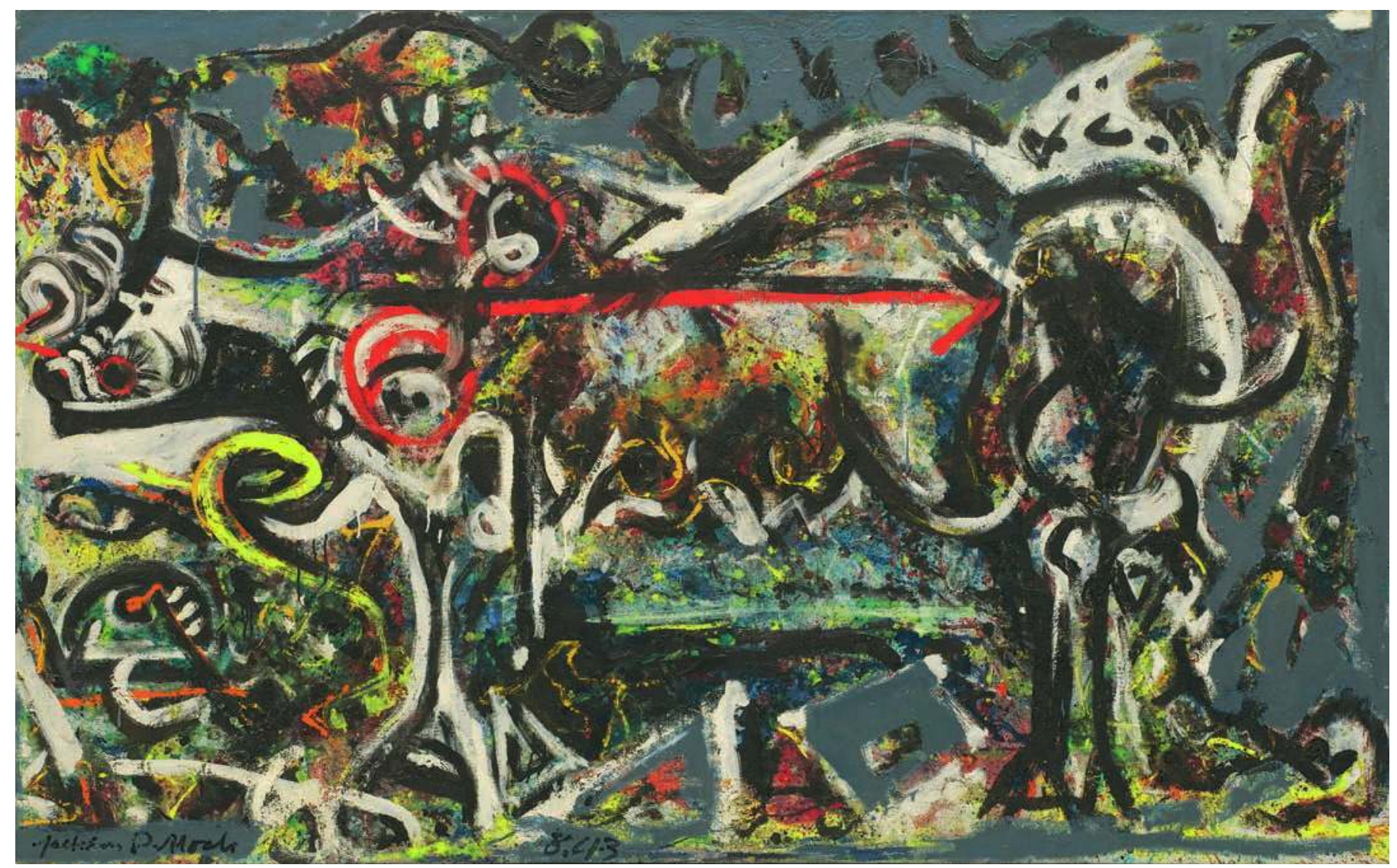

Fig. 10 Jackson Pollock, La Louve, 1943, MoMA, New York / Scala, Florence @ 2011 Pollock-Krasner Foundation / ADAGP, Paris 2011. 


\section{CARL EINSTEIN ET LES PRIMITIVISMES}

- -

70. Carl Einstein, «Die Kolonne Durruti » (novembre 1936), Werke, t. III, op. cit., p. 520 ; traduction infra, p. 253.

71. Id., Die Fabrikation der Fiktionen, op. cit., p. 327.

72. De longs extraits de ses notes préparatoires sont publiés dans Werke, t. IV, op. cit., p. 301-447; traduction française in Cahiers du musée national d'Art moderne 117, 2011.

73. Carl Einstein, Werke, t. IV, op. cit., p. 304, 308, 309, 312, 31 ?

74. «Les images », ajoutait Einstein, étaient «partiellement actives sur un mode animiste - une position qui, avec le déclin de l'animisme, devint intenable; autrement dit, [elles] furent rabaissées au rang de représentations. » Ibid., p. 370, 371. 75. Ibid., p. 370. la force du travail anonyme. L'anonymat et le communisme ne font qu'un ${ }^{70}$." À en croire ce témoignage, c'est dans la guerre civile espagnole qu'Einstein put finalement connaître, fût-ce brièvement, l'expérience collective qu'il avait en vain recherchée dans la révolution allemande et qu'il avait par suite espéré pouvoir réaliser à travers l'art.

Quel rôle l'art pouvait-il encore jouer à présent? Einstein avait déjà compris qu'il pouvait, au mieux, remplir une fonction utile pour la société " en collaborant modestement à la production d'une nouvelle réalité ", ce qui allait de pair avec un renoncement à son statut privilégié et aux illusions d'une « utopie séparée ${ }^{71}$ ». Cependant, l'abandon des espoirs qu'il avait placés dans le modernisme n'impliquait pas, pour Einstein, celui de ses écrits sur l'art. Après son retour à Paris, au début de l'année 1939, il recommença à travailler sur son projet inachevé, extrêmement ambitieux, d'un Manuel de l'art (Handbuch der Kunst), qui devait, à en croire un plan retrouvé dans ses archives, comprendre cinq volumes ${ }^{72}$. Si, dans La Fabrication des fictions, Einstein réglait ses comptes avec ses propres illusions modernistes, le Manuel et un autre projet tardif inachevé, le Traité de la vision, peuvent être vus comme une tentative pour repenser l'art et son histoire, et pour replacer à l'échelle de cette dernière ce qu'il considérait désormais comme l'échec du projet de la modernité. Dans les quatre dossiers de notes qui peuvent être datés avec certitude de la période postérieure à son retour d'Espagne, les thèses audacieuses et passionnées d'Einstein avaient cédé la place à une recherche dont l'issue demeurait ouverte, et à une modestie sans précédent chez lui.

Le plan détaillé du Manuel de l'art montre que celui-ci était censé retracer, en partant de l'époque paléolithique, l'évolution de l'art depuis le temps où il se définissait comme un mode d'expression collectif, sur une base large, jusqu'au déclin qui l'avait réduit, à l'époque moderne, à un statut élitiste et individualiste ${ }^{73}$. Dans l'un des derniers dossiers qui contenaient ses réflexions ultimes sur le sujet, Einstein évoquait cette décadence du pouvoir de l'art:

Dès qu'on a représenté de façon consciente - autrement dit, dès qu'on a établi une séparation entre l'être et l'image, l'art est devenu plus indirect, plus élastique et plus arbitraire -, les œuvres d'art ont perdu de leur signification et, évidemment, leur effet est devenu moins profond: les œuvres d'art n'étaient plus que des représentations, ce n'étaient plus des personnes dans un état particulier (morts) ni des forces - au demeurant, les œuvres d'art se distinguaient du dieu ou de la personne en ce qu'elles n'abritaient que des états ou des forces partiels, des parties, pour ainsi dire, et ne possédaient donc pas la complexité du phénomène «vivant ». [...]

À partir de là, perte de la force des images ${ }^{74}$.

Einstein songeait ici à la pratique animiste qu'il avait pour la première fois décrite dans Negerplastik - «[L'œuvre d'art] ne signifie rien, elle n'est pas symbole; elle est le dieu "-comme le sine qua non de toute effectivité sociale de l'art. Nous l'avons vu, il avait tenté - passionnément, désespérément, aveuglément - de retrouver dans le cubisme et le surréalisme l'image animiste de l'art africain, et cette tentative s'était soldée par un échec complet. Après avoir déclaré que l'art déterminait la perception humaine et définissait notre réalité visuelle, il reconnaissait à présent l'existence d'une différence fondamentale entre être et image, et concédait : "La représentation est distincte de la vision ${ }^{75}$. " Pour lui, ce constat impliquait la perte irréversible de l'agentivité qu'il avait auparavant attribuée à l'art.

Dans ses notes pour le Traité de la vision, Einstein en appelait désormais à des recherches empiriques, des «travaux de laboratoire» destinés à étudier les 
réponses de sujets humains à des questions portant sur des domaines tels que la perception, la mémoire visuelle, les réactions à des formes ${ }^{76}$. Le contraste ne pouvait être plus accusé avec les certitudes du livre sur Braque et ses utopies irréalistes relatives aux réactions du spectateur de l'œuvre d'art. Cette investigation rationnelle, au demeurant, n'était pas sans présenter un aspect mélancolique : «La tentative pour préciser les effets de l'art, observait Einstein, était peut-être un signe - le signe que nous avons déjà pris congé de lui7. »

Williams College, Department of Art Charles.W.Haxthausen@williams.edu

Traduit de l'anglais et de l'allemand par Isabelle Kalinowski
- $\bullet$

76. Ibid., p. 259-265.

77. Carl Einstein, «Handbuch der Kunst », CEA, microforme 242, p. 14.

mots clés / keywords : agentivité // agency • collectivité // collectivity • Afrique //

Africa • cubisme // cubism • surréalisme // surrealism.

\section{Résumé / Abstract}

Charles W. Haxthausen, Art, agentivité et collectivité - Dès le début des années 1930, Carl Einstein crut passionnément que l'art moderne avait le pouvoir de changer radicalement le monde visuel de l'homme et, par là même, de transformer la subjectivité humaine et notre construction de la réalité. Pourtant, tout en invoquant de manière récurrente la « collectivité », il demeurait vague quant à la nature précise de «l'utopie révolutionnaire » à laquelle la forme artistique était appelée à donner le jour. Comment, au juste, la forme artistique pouvait-elle réaliser une telle utopie? Comment pouvait-elle générer - puisque c'est là ce qu'Einstein semblait croire - des formes sociales et politiques particulières? Un monde visuel collectif informé par l'art et manifesté dans un style unifié pouvait-il mener à une société égalitaire ? Einstein ne clarifia ce point qu'en abandonnant sa croyance dans l'agentivité sociale de l'art moderne, ce qui eut pour corrélat une révision radicale de son attitude vis-à-vis de l'avant-garde. L'article résume les étapes majeures de cette odyssée intellectuelle et insiste sur la place centrale de l'art africain dans l'élaboration des conceptions d'Einstein.
Charles W. Haxthausen, Art, Agency, and Collectivity - Until the early 1930s Carl Einstein believed passionately in modern art's potential to radically alter human visuality and in so doing to alter human subjectivity and our construction of reality. Yet, beyond recurrent references to "collectivity", he was vague about the precise nature of the "revolutionary utopia" that artistic form was to bring about. How exactly might artistic form achieve that utopia? How could it generate-for that is what Einstein had apparently believed-particular social and political forms? Would a collective visuality, shaped by art and manifest in a unified style, lead to an egalitarian collective society? Clarity in these matters came only with Einstein's abandonment of his belief in modern art's social agency, resulting in a radical revision of his attitude toward the avant-garde. This article plots the major stages of this intellectual odyssey and argues for the centrality of African art in shaping Einstein's views. 